\title{
CUIDANDO DE QUEM CUIDA EM TEMPOS DE PANDEMIA: UM RELATO DE EXPERIÊNCIA ${ }^{1}$
}

\author{
TAKING CARE OF THOSE WHO CARE IN PANDEMIC TIME: \\ AN EXPERIENCE REPORT
}

\author{
Stefani Gabriela da Silva dos Santos², Fernanda Lencina de Amarante ${ }^{3}$, \\ Ana Paula Brutti Linhatti ${ }^{4}$, Suani Feltrin da Silva ${ }^{5}$, \\ Joyce Nagera Braz ${ }^{6}$ e Felix Miguel Nascimento Guazina ${ }^{7}$
}

\section{RESUMO}

Objetivos: este estudo trata-se da vivência de residentes de: Enfermagem, Fisioterapia, Nutrição, Psicologia e Terapia Ocupacional, e refere-se a atendimentos voltados à saúde dos trabalhadores de um hospital de médio porte da região central do Rio Grande do Sul, frente à pandemia da COVID-19. Metodologia: esta pesquisa apresenta uma abordagem descritiva e se caracteriza como um relato de experiência referente aos serviços prestados pelas residentes, de abril a julho de 2020. Resultados: nesse tempo, foram realizados 305 atendimentos individuais, considerando o acesso de 74 trabalhadores, sendo, em sua maioria, técnicos de enfermagem e enfermeiros das unidades de internação clínica, com predomínio do sexo feminino. Dentre os motivos pelos quais os trabalhadores buscaram atendimento do projeto, destacam-se: a ansiedade e a preocupação com o risco de contaminação, e o medo da transmissão do vírus para os familiares oriundos da nova realidade vivida com a COVID-19. Ainda, foram percebidas queixas de dores relacionadas à rotina de trabalho, como a sobrecarga de serviço, falta de planejamento ergonômico e a ocorrência de lesões adquiridas, bem como de compulsão alimentar, sobrepeso e sintomas gástricos gerados pela ansiedade do trabalho em tempo de pandemia, associados à falta de tempo para a prática de atividade física. Conclusão: concluiu-se que os atendimentos aos trabalhadores propiciaram uma melhora na sua qualidade de vida, auxiliando-os no processo de enfrentamento da COVID-19. Também, possibilitou o aperfeiçoamento das residentes no que concerne à conduta profissional em situações de emergência e desastre, como a realidade da pandemia.

Palavras-chave: Assistência Hospitalar; COVID-19; Saúde do Trabalhador.

\section{ABSTRACT}

Objectives: this study deals with the experience of residents of: Nursing, Physiotherapy, Nutrition, Psychology and Occupational Therapy, and refers to health care for workers in a medium-sized hospital in the central region of Rio Grande do Sul, against the COVID-19 pandemic. Methodology: this research presents a

1 Trabalho de Residência Multiprofissional em Saúde.

2 Autor, Psicóloga, Residente em Reabilitação física - Universidade Franciscana (UFN). E-mail: sgsssantos@ucs.br

3 Coautor, Terapeuta Ocupacional, Especialista em Sistema Público de Saúde - Universidade Federal de Santa Maria (UFSM), Residente em Reabilitação física - Universidade Franciscana (UFN). E-mail: amarante.fer@gmail.com

4 Colaborador, Fisioterapeuta, Residente em Reabilitação física - Universidade Franciscana (UFN). E-mail: ana.paula.brutti@ hotmail.com

5 Colaborador, Nutricionista, Especialista em Nutrição Clínica e Metabolismo - Colégio Brasileiro de Estudos Sistêmicos (CBES), Residente em Reabilitação física - Universidade Franciscana (UFN). E-mail: suani.feltrins@gmail.com 6 Colaborador, Enfermeira, Especialista em Enfermagem no Trabalho - Uninter, Residente em Reabilitação física Universidade Franciscana (UFN). E-mail: joycenagera@gmail.com

7 Orientador, Psicólogo, Doutor em Psicologia Social, Tutor no Programa de Residência Multiprofissional em Reabilitação Física - Universidade Franciscana(UFN). E-mail: guazina@gmail.com 
descriptive approach and is characterized as an experience report referring to the services provided by residents, from April to July 2020. Results: in that time, 305 individual consultations were carried out, considering the access of 74 workers, most of whom were nursing technicians and nurses from clinical inpatient units, with a predominance of females. Among the reasons why workers sought care for the project, the following stand out: anxiety and concern about the risk of contamination, and fear of transmission of the virus to family members from the new reality experienced with COVID-19. Still, complaints of pain related to the work routine were perceived, such as overload of service, lack of ergonomic planning and the occurrence of acquired injuries, as well as binge eating, overweight and gastric symptoms generated by work anxiety in a pandemic time, associated with lack of time to practice physical activity. Conclusion: it was concluded that the care provided to workers provided an improvement in their quality of life, assisting them in the process of coping with COVID-19. It also made it possible for residents to improve their professional conduct in emergency and disaster situations, such as the reality of the pandemic.

Keywords: Hospital Care; Coronavirus Infections; Occupational Health.

\section{INTRODUÇÃO}

Segundo o Ministério da Saúde (MS), a COVID-19 é uma doença causada pelo Coronavírus, em que o portador pode apresentar infecções assintomáticas - cerca de $80 \%$ dos casos e até mesmo quadros graves de dificuldade respiratória, demandando de atendimento hospitalar de alta complexidade (BRASIL, 2020a). O espectro clínico desta infecção também pode ser de doença leve do trato respiratório superior, pneumonia viral grave com insuficiência respiratória, acarretando até mesmo em morte (ZHOU, 2020).

Em março de 2020, a Organização Mundial de Saúde (OMS) caracterizou essas frequentes transmissões como uma pandemia. No Brasil, até julho de 2020, foram registrados cerca de 14.562.550 casos de COVID-19, dentre estes, 607.781 evoluíram a óbito conforme dados da Organização Pan-Americana de Saúde (OPAS, 2020).

Como forma de contenção e combate a esse vírus, o MS regulamentou estado de quarentena em todo o Brasil, sendo definida pelo isolamento social até que se reduza o risco de contaminação, medida esta que se caracteriza pelo comportamento em que o indivíduo deixa de participar voluntariamente ou não do convívio com o restante da sociedade, abstendo-se de participar de atividades sociais em grupo. Essa é uma medida de precaução integrante das ações para enfrentamento da Emergência em Saúde Pública de Importância Internacional (ESPII), proveniente do Coronavírus (SCHNEIDERS; PACHECO, 2020). Situação que já ultrapassa os 12 meses de ocorrência no Brasil.

A pandemia avançou abruptamente, atingindo diversos países, estados e cidades, trazendo consigo não só impactos econômicos, sociais e físicos, mas, também, emocionais. Estudos recentes ressaltam o quão impactante é essa pandemia para a saúde mental da população em geral, tendo afetando também para os profissionais da saúde, resultante de diversos temores como medo da exposição ao contágio, à situação do isolamento e as medidas de quarentena (CRUZ et al., 2020). 
Como consequência desse abalo psicológico, pode-se perceber, nos trabalhadores, a redução de: empatia, exaustão, ansiedade, irritabilidade, insônia e, também, redução de desempenho assertivo nos campos de trabalho. Ainda, percebe-se o aumento de Transtornos Mentais Comuns (TMC), como a fadiga e a agressividade, além de estresse agudo, episódio de pânico, manifestação de preditores de Estresse Pós-Traumático (TEPT), depressão e ansiedade (CRUZ et al., 2020).

Zwielewski et al. (2020) alegam que a quarentena pode ser vivenciada e interpretada de diversas formas, o que vai depender de inúmeros fatores, como as condições socioeconômicas do sujeito, bem como suas características de personalidade. Frequentemente, ela pode ser experienciada de forma desagradável, desconfortável e/ou dolorosa. A situação exige separação e, ao mesmo tempo, confinamento, ocorrendo suspensão de rituais de lutos e despedidas, podendo acarretar sentimentos como de impotência, falta de liberdade, solidão, tédio, incertezas, raiva, medo, entre outros. As medidas de restrições afetam, também, a dinâmica laborativa e familiar, uma vez que ocorre o fechamento de empresas, escolas e locais públicos, demandando uma reorganização e exigindo um movimento imediato por parte dos sujeitos. Ainda, este autor a traz que o contexto atual resultante da COVID-19 pode ser encarado como uma oportunidade de criação e desenvolvimentos de serviços de orientação, atenção e tratamento à saúde mental e física dos envolvidos nesse processo, como é o caso dos profissionais da Saúde atuantes que estão acentuadamente propensos ao abalo consequente da pandemia, uma vez que estão na linha de frente de combate e tratamento, aumentando o risco de contaminação biológica e letalidade nesses profissionais (ZWIELEWSKI et al., 2020).

Pensando no atual cenário vivido pelos indivíduos em decorrência da COVID-19, bem como em todas as consequências geradas, por essa doença, em diferentes esferas da vida, percebeu-se a necessidade de criar um espaço de atendimento voltado aos funcionários atuantes em um hospital de baixa e média complexidade no interior do Estado do Rio Grande do Sul. Dessa forma, o objetivo da atuação das residentes de: Enfermagem, Fisioterapia, Nutrição, Psicologia e Terapia Ocupacional neste projeto foi o de proporcionar acolhimento e melhorias na qualidade de vida dos trabalhadores, auxiliando assim no cotidiano vivenciado atualmente devido a COVID-19. Assim sendo, este trabalho foi delineado buscando evidenciar, por meio de um relato, a experiência destas profissionais.

\section{MÉTODO}

Este estudo tem uma abordagem descritiva e se caracteriza como um relato de experiência. Este busca propiciar reflexões sobre a prática dos autores deste artigo na assistência dos trabalhadores da área da Saúde, com vistas à ao referencial teórico acessado, uma vez que o relato de experiência descreve, em um texto acadêmico, uma determinada experiência, com o intuito de contribuir em uma área de atuação (FLICK, 2008). 
Assim sendo, retrata a vivência de residentes do Programa de Residência Multiprofissional em Reabilitação Física, dentre os meses de abril a julho de 2020, compreendendo os núcleos profissionais: Enfermagem, Fisioterapia, Nutrição, Psicologia e Terapia Ocupacional, atuantes em um projeto voltado à saúde dos trabalhadores de um hospital de médio porte situado no interior do estado do Rio Grande do Sul.

As vivências foram registradas por meio de um relatório de práticas, objetivando descrever as percepções e entendimentos frente às demandas trazidas por cada funcionário que buscou atendimento no referido projeto. Optou-se por realizar o estudo em formato de relato de experiência, já que se tem como foco abordar questões percebidas e vivenciadas durante a atuação profissional, visando à troca de conhecimentos e manejo no atual momento da COVID-19.

\section{RESULTADOS E DISCUSSÃO}

\section{O PROJETO - SAÚDE DO TRABALHADOR DIANTE DA COVID-19}

Durante a rotina no referido hospital deste estudo, observou-se um aumento significativo de funcionários com atestados ou afastamento das atividades laborais, além da nitidez de dificuldades enfrentadas com o manejo da situação da pandemia, gerando aumento de sintomas de ansiedade e pressões psicológicas. Com base nisso, o Serviço Especializado em Engenharia de Segurança e Medicina do Trabalho (SESMT), em parceria com as residentes alocadas no hospital, estruturam tal projeto, que possui como principal finalidade prestar assistência aos funcionários da instituição visto a vivência de um momento difícil e turbulento devido ao cenário atual causado pela COVID-19.

Quando alinhadas as intervenções a serem realizadas entre os idealizadores e a coordenação do hospital, iniciou-se o processo de divulgação do projeto junto aos colaboradores, e, de modo presencial, foi explanado em cada setor sobre a proposta, os dias e local de atendimentos. Feito isso, logo foram enviadas mensagens de texto via celular nos grupos de aplicativo existente entre as equipes, bem como disponibilizados cartazes, objetivando divulgar o projeto em cada um dos setores que constituem o hospital, desde a assistência de internação clínica ou cirúrgica ao administrativo e relembrar os funcionários da existência dele.

O projeto em questão ocorreu em dois turnos semanais - manhã e tarde - entre os meses de abril e junho de 2020, no qual aconteceu um acolhimento multiprofissional de caráter humanizado, em que foram explicados seus objetivos, seguido de uma Anamnese com dados sociodemográficos, condições de saúde e principais queixas referidas por cada funcionário que buscou assistência; após, os funcionários foram encaminhado para serem acompanhados pelas residentes das áreas de Enfermagem, Fisioterapia, Nutrição, Psicologia e Terapia Ocupacional, para atendimento individual ou 
interprofissional, de acordo com suas demandas. Até o momento, 74 trabalhadores do hospital participaram da assistência proposta pelo projeto, totalizando 305 atendimentos.

No decorrer deste projeto, percebeu-se que a frequência maior de participantes são os trabalhadores da assistência direta ao paciente, com predomínio do sexo feminino (92\%), em sua maioria técnicas de enfermagem das unidades de internação clínica, seguido de enfermeiras. Apenas 8\% são participantes homens, uma questão bastante notada também nos serviços de saúde em geral.

Consoante a isso, no estudo de Gomes, Nascimento e Araújo (2007), que investiga a procura de serviços de saúde por sujeitos do sexo masculino, os autores afirmam que o imaginário do homem, atrelado às questões culturais de que ele poderia vir a parecer fraco, vulnerável e menos viril, o que dificulta a adoção de práticas preventivas e de autocuidado por ele. Tais autores concluem que a organização dos serviços não estimula essa busca, bem como não incentiva isso por meio de campanhas de saúde pública. No decorrer do projeto, foi percebida a ausência dos profissionais de apoio, como dos setores de higienização, nutrição, lavanderia e farmácia, mesmo após a divulgação intensificada do projeto. Tal fato possivelmente pode estar relacionado ao baixo número de colaboradores nesses setores, em contrapartida à demanda de serviço, podendo também ser influenciado pela não liberação dos trabalhadores pelos gestores internos.

Com base na procura dos funcionários ao serviço oferecido, pode-se pensar na realidade de adoecimentos muitas vezes vivenciada pelos profissionais da área da Saúde, uma vez que estes estão sujeitos a serem prejudicados por complicações físicas, psicológicas, podendo vir a adoecer por diversos aspectos relacionados ao trabalho. No que tange a pouca procura por profissionais das áreas de apoio, é possível pensar que o ato de adoecer, ou até mesmo a procura por ajuda ou auxílio profissional, pode ser percebida como sinal de fraqueza e vergonha, pois demanda interrupção do trabalho para a busca de ajuda (BROTTO; DALBELLO-ARAÚJO, 2012).

Dentre os motivos pelos quais os trabalhadores buscaram atendimentos no projeto, destacaram-se: ansiedade, preocupação, apreensão, insegurança e constante medo, oriundos da nova realidade vivida por causa da COVID-19. Dentre os relatos, foram notadas dúvidas relacionadas ao risco de contaminação no ambiente hospitalar, ao uso dos Equipamentos de Proteção Individual (EPIs), além de medo evidente da transmissão do vírus para os familiares e pessoas de seu convívio. A prevalência de exaustão física e mental é nítida entre os profissionais da saúde, além do aumento recorrente de ansiedade, depressão, perda da qualidade do sono, aumento do uso de drogas, sintomas psicossomáticos, fora a dor pela perda de pacientes e colegas, e a preocupação da possibilidade de infecção e transmissão para familiares (MEDEIROS, 2020). Devido à recente descoberta da disseminação do vírus, ainda são poucos os dados sobre o abalo na saúde mental da população causado pela pandemia, mas, os que existem, são alarmantes, indicando, assim, a importância da implementação de meios para redução de estresse, ansiedade, insônia, tristeza, mágoa, desamparo, depressão, frustração e, até mesmo, culpa, tanto pelo fato da perda de algum paciente, como, também, da possibilidade de contaminação de entes queridos (ZWIELEWSKI et al., 2020). 
Outra consideração observada durante a atuação das autoras foi o quanto a influência do fechamento dos setores de beleza e bem-estar para a realização do autocuidado, como das clínicas de fisioterapia e Pilates, além dos salões de beleza e de manicure interfere no humor dos profissionais, assim como se torna um empecilho para um enfrentamento mais positivo da situação atual, uma vez que a tendência é se fusionar mais ao problema e suas consequências. Ainda foram identificados relatos que expressam desconforto por dores físicas, estas relacionadas à rotina como: a falta de planejamento ergonômico dos postos de trabalho, a sobrecarga de serviço - muitos pacientes para poucos profissionais - e a ocorrência de lesões adquiridas, como a Lesão por Esforço Repetitivo (LER), Distúrbio Osteomuscular Relacionado ao Trabalho (DORT), além da contratura muscular devido à tensão emocional e ao estresse explícito vivenciado. Segundo o MS (BRASIL, 2019), LER e DORT são as doenças que mais afetam os trabalhadores brasileiros, não apenas os da área da Saúde, com um aumento de $184 \%$ em 10 anos, indicando que há maior exposição de trabalhadores a fatores de risco que podem ocasionar na incapacidade funcional.

Ademais, reparou-se a procura por atendimento devido à compulsão alimentar, excesso de peso e sintomas gástricos gerados pela ansiedade do trabalho em tempos de pandemia, associados à falta de tempo para a prática de atividade física e, até mesmo, a não disponibilização de espaços disponíveis para isso, em consequência do isolamento social. Consoante isso, segundo o estudo de Hidalgo et al. (2016), as condições de trabalho como longas jornadas demandas excessivas e um ambiente hostil contribuem para a prevalência de obesidade entre os trabalhadores, pois pode influenciar o estilo de vida e os hábitos alimentares, afetando a saúde como um todo. Os autores ainda evidenciam que existe relação entre o estresse emocional e a adoção de alguns comportamentos alimentares, de acordo com a vulnerabilidade de cada sujeito frente a esse estresse. Diante disso, poderá surgir tanto um aumento como uma restrição na ingestão alimentar. Além disso, a compulsão alimentar por curtos períodos de tempo também é observado em momentos de sofrimento psicológico (OLIVEIRA et al. 2020).

No desenvolver do projeto aqui apresentado, validou-se a necessidade de uma atenção multiprofissional ao trabalhador, não somente no momento atual, mas cotidianamente, lançando mão de um olhar atento para a integralidade do trabalhador, pois este é um sujeito biopsicossocial. Assim sendo, o trabalho multiprofissional em equipe surge como forma de alcançar o cuidado integral em saúde, atingindo aspectos curativos, preventivos, de reabilitação, incluindo o indivíduo e seu contexto, além de que a realidade do trabalhador é complicada, já que, muitas vezes, não são consideradas as características individuais (PEREIRA; RIVERA; ARTEMANN, 2013).

Essa assistência foi prestada mediante intervenções, como: acolhimento com escuta qualificada, legitimando e validando todos os sentimentos e emoções geradas pela atual realidade, além de se disponibilizar para escutar as percepções de cada funcionário atendido quanto ao enfrentamento da pandemia e suas consequências; e aferição dos sinais vitais para verificação dos valores de referência habituais, visto que o estresse e a ansiedade geram sintomas psicológicos e físicos, tais como calafrios, 
batimentos cardíacos acelerados, aumento do suor, boca seca, formigamentos e cólicas abdominais (SOUZA, 2017). Cabe ressaltar que, conforme estudos se pode verificar que emoções como a ansiedade, quando bloqueadas, favorecem a crise hipertensiva em pacientes que possuem predisposição genética (FONSECA et al., 2009).

Para atuar sobre os aspectos de saúde que interferem no desempenho ocupacional dos trabalhadores, também foi disponibilizado o acompanhamento com a Auriculoterapia, previsto pelo MS no conjunto de Práticas Integrativas e Complementares em Saúde (BRASIL, 2020b). Ainda, foram realizadas avaliações e orientações nutricionais para as principais comorbidades referidas pelos servidores, bem como intervenções corporais de alongamento, relaxamento, liberação miofascial e fortalecimento muscular, de acordo com a demanda de cada servidor.

Em toda intervenção realizada, ocorreu o emprego de técnicas próprias dos profissionais envolvidos, levando em consideração a importância da humanização da saúde. Considerou-se que a atuação profissional é influenciada por essa integralidade demandando atenção voltada para a subjetividade frente a cada situação vivenciada em sua atividade laboral, assim como relacionamento interpessoal.

De acordo com Sato, Lacaz e Bernardo (2006), as questões que norteiam o trabalho vão além dos aspectos biológicos, envolvendo o social e o psíquico através de um olhar que inclui ações de prevenção, assistência e promoção da saúde. Para Heloani e Lancman (2004), é necessária a compreensão dos processos que permeiam o trabalho para que se possa pensar intervenções in loco visando a minimizar as diversas fontes geradoras de sofrimento e otimizar as prazerosas, a fim de se pensar na transformação das organizações. Quanto a esse viés de transformação, Clot (2011) afirma que um ambiente saudável de trabalho é possível a partir de espaços que viabilizam contextos e vivências de modo a desenvolver, no trabalhador, a alteridade e a autonomia sobre o mundo do trabalho e sobre si mesmo.

A percepção frente à atuação das autoras nesse projeto - tanto no espaço de escuta protegido durante os atendimentos, quanto em diálogo junto às residentes nas unidades de trabalho, local onde elas também atuam - notou-se melhora em relação a algumas demandas levadas aos atendimentos, principalmente no que diz respeito aos sentimentos de estresse, medo, e os que envolvem ansiedade, além de um alívio de sintomas físicos, ocasionando em um melhor manejo da situação atual por parte dos funcionários. Além de um impacto positivo nas relações interpessoais entre os profissionais da assistência, como enfermeiros e técnicos de enfermagem que mantiveram acompanhamento, de modo que possivelmente oportunizou a redução de estresse e a ansiedade. Ademais, percebeu-se que a demanda por atendimentos multiprofissionais vai além dos funcionários, dado que muitos destes solicitaram atendimentos/acompanhamento para seus familiares, sobre os quais identificaram a necessidade de intervenções oferecidas pelo projeto.

Com base nisso tudo, em momento de pandemia, destaca-se fortemente a necessidade de um suporte emocional e assistência para a saúde mental de toda a população, já que é um fato que 
abalou e está abalando toda população mundial de forma geral, não apenas os profissionais da Saúde. Atualmente, está sendo vivenciado um momento de crise e emergência, com reflexos na sociedade, na economia e na saúde física e mental das populações, salientando a necessidade de promoção de ações voltadas para minimizar e assistir tais impactos (CRUZ et al., 2020).

\section{CONCLUSÃO}

Com base neste estudo e no trabalho realizado, pode-se perceber o quão impactante é o momento atual vivenciado em todo o mundo, influenciando, de diversas formas e maneira, a vida de cada indivíduo, principalmente daqueles que estão na linha da frente para auxiliar a combater tal pandemia. Em virtude de ser um momento único e atípico, foram manejadas e vivenciadas situações nunca antes experimentadas, principalmente em se tratando do suporte oferecido e realizado a outros indivíduos frente a uma pandemia, exigindo, assim, dos profissionais atuantes no projeto, um preparo teórico para lidar com tais circunstâncias.

As atividades desenvolvidas e oferecidas aos funcionários pela instituição objetivaram propiciar uma melhora na qualidade de vida destes e estratégias mais adaptáveis, auxiliando-os no processo de enfrentamento dessa situação atípica vivenciada atualmente por causa da COVID-19. Através da estrutura e desenvolvimento deste estudo, baseado na atuação profissional das autoras, pode-se relatar os benefícios alcançados a partir da assistência diferenciada e personalizada aos trabalhadores da saúde, buscando assim transmitir tais vivências para os demais profissionais interessados na temática.

As demandas abordadas durante os atendimentos mostraram a necessidade de dar continuidade ao projeto mesmo após a pandemia, focando na assistência integral desses funcionários, sendo este indispensável para uma assistência digna ao paciente não apenas em momento de pandemia. Sendo assim, este projeto possibilitou melhores aprofundamentos sobre a necessidade e importância de serviços voltados para os trabalhadores da saúde, assim como vislumbrar algumas das possíveis atividades a serem desenvolvidas em prol desse objetivo, tanto neste quanto em outros serviços hospitalares. Propiciou, ainda, o aperfeiçoamento das residentes envolvidas no projeto quanto à conduta profissional em situações de emergência e desastre, como a realidade da pandemia.

\section{REFERÊNCIAS}

BRASIL. Ministério da Saúde. Secretaria de Vigilância em Saúde. Departamento de Vigilância de Doenças e Agravos não Transmissíveis e Promoção da Saúde. Saúde Brasil 2018 uma análise de situação de saúde e das doenças e agravos crônicos: desafios e perspectivas. Brasília: Ministério da Saúde, 2019. 424 p. Disponível em: https://bit.ly/3vad5tx. Acesso em: 2 jul. 2020. 
BRASIL. Ministério da Saúde. O que é COVID-19. 2020a. Disponível em: https://bit.ly/3eiOhc2. Acesso em: 27 jul. 2020.

BRASIL. Ministério da Saúde. Práticas integrativas e complementares (PICS): quais são e para que servem. 2020b. Disponível em: https://bit.ly/3enLpKX. Acesso em: 28 jul.2020.

BROTTO, T.C.; DALBELLO-ARAUJO, M.. É inerente ao trabalho em saúde o adoecimento de seu trabalhador. Revista Brasileira de Saúde Ocupacional, São Paulo , v. 37, n. 126, p. 290-305, Dez. 2012. Disponível em: https://doi.org/10.1590/S0303-76572012000200011.

CLOT, Y. Clínica do trabalho e clínica da atividade. In: BENDASSOLLI, Pedro F.; SOBOLL, Lis A. P. (Org.). Clínicas do trabalho: novas perspectivas para compreensão do trabalho na atualidade. São Paulo: Atlas, 2011. p. 3-16.

CRUZ, R.M. et al. COVID-19: Emergência e Impactos na Saúde e no Trabalho. Revista Psicologia Organizações e Trabalho, Florianópolis, v. 20, n. 2, p. 1-3, abr-jun. 2020. Disponível em: http:// dx.doi.org/10.17652/rpot/2020.2.editorial.

FLICK, U. Uma introdução à pesquisa qualitativa. 3. ed. Porto Alegre: Bookman, 2008. 408p.

FONSECA, F.C. et al. A influência de fatores emocionais sobre a hipertensão arterial. Jornal Brasileiro de Psiquiatria, Rio de Janeiro, v. 58, n. 2, p. 128-134, 2009. Disponível em: https://doi.org/10.1590/ S0047-20852009000200011.

GOMES, R.; NASCIMENTO, E.F.; ARAÚJO, F.C. Por que os homens buscam menos os serviços de saúde do que as mulheres? Caderno de Saúde Pública, Rio de Janeiro, 23(3):565-574, mar, 2007.

HELOANI, R.; LANCMAN, S. Psicodinâmica do trabalho: o método clínico de intervenção e investigação. Produção, São Paulo, v. 14, n. 3, p. 77-86, dez. 2004. Disponível em: https://doi.org/10.1590/ S0103-65132004000300009.

HIDALGO, K.D. et al. Health promoting practices and personal lifestyle behaviors of Brazilian health professional. Biomed Central Public Health, v. 16, n.1114, 2016. Disponível em: http://dx.doi.org/ 10.1186/s12889-016-3778-2. 
MEDEIROS, E.A. A luta dos profissionais de saúde no enfrentamento da COVID-19. Acta Paulista de Enfermagem, v. 33, 2020.

OLIVEIRA, A. et al. COVID-19: Comportamentos alimentares e outros estilos de vida saudáveis em tempo de isolamento social. In. Da emergência de um novo vírus humano à disseminação global de uma nova doença - Doença por Coronavírus 2019 (COVID-19). Disponível em: https://bit.ly/3xfhahP. Acesso em 25 Set 2020.

ORGANIZAÇÃO PAN-AMERICANA DA SAÚDE (OPAS). Folha informativa - COVID-19 - doença causada pelo novo coronavírus. 2020. Disponível em: https://bit.ly/2QIpC8G. Acesso em: 27 jul. 2020.

PEREIRA, R.C.; RIVERA, F.J.; ARTMANN, E. O trabalho multiprofissional na estratégia saúde da família: estudo sobre modalidades de equipes. Interface (Botucatu), Botucatu, v. 17, n. 45, p. 327-340, 2013. Disponível em: https://doi.org/10.1590/S1414-32832013005000006.

SATO, L.; LACAZ, F.A.; BERNARDO, M.H. Psicologia e saúde do trabalhador: práticas e investigações na Saúde Pública de São Paulo. Estudos de psicologia (Natal), v. 11, n. 3, p. 281-288, dez. 2006. Disponível em: https://doi.org/10.1590/S1413-294X2006000300005.

SCHNEIDERS, L.; PACHECO, S. Saúde regulamenta condições de isolamento e quarentena. 2020. Ministério da Saúde (Brasil). Disponível em: https://bit.ly/3eo2TqD. Acesso em: 27 jul. 2020.

SOUZA, D.C. Condições emocionais de estudantes universitários: estresse, depressão, ansiedade, solidão e suporte social. 2017. 90 f. Dissertação (Mestrado) - Curso de Mestrado em Psicologia, Programa de Pós-Graduação em Psicologia, Universidade Federal do Triângulo Mineiro, Uberaba, 2017. Disponível em: http://bdtd.uftm.edu.br/handle/tede/507. Acesso em: 5 jul. 2020.

Zhou, F. et al. Clinical course and risk factors for mortality of adult inpatients with COVID-19 in Wuhan, China: a retrospective cohort study. The Lancet. Vol 395. March 28, 2020. Disponível em: https://doi.org/10.1016/s0140-6736(20)30566-3.

ZWIELEWSKI, G. et al. Protocolos para tratamento psicológico em pandemias: as demandas em saúde mental produzidas pela COVID-19. Revista Debates em Psiquiatria, n. 2, abr/jun, p. 30-37, 2020. 